\title{
The Method of Interacting Configurations in Complex Number Representations: From Helium to the Complex Atoms
}

\author{
V.M. Simulik ${ }^{1, *}$, R.V. Tymchyk ${ }^{1}$, T.M. Zajac ${ }^{2}$ \\ ${ }^{1}$ Institute of Electron Physics, National Academy of Sciences, Uzhgorod, 88000, Ukraine \\ 2 Department of Electronic Systems, Uzhgorod National University, Uzhgorod, 88000, Ukraine \\ *Corresponding Author: vsimulik@gmail.com
}

Copyright (C) 2017 by authors, all rights reserved. Authors agree that this article remains permanently open access under the terms of the Creative Commons Attribution License 4.0 International License

\begin{abstract}
The transition from the He atom to the complex atoms description in the method of interacting configurations in the complex number representation has been presented. As a first step the problem of ionization of $\mathrm{H}^{-}$and $\mathrm{Li}^{+}$ions is considered. The spectroscopic characteristics of the Be, $\mathrm{Mg}$ and $\mathrm{Ca}$ atoms in the problem of the electron-impact ionization of these atoms are investigated. Few results in the photoionization problem on the ${ }^{1} P$ autoionizing states above the $\mathrm{n}=2$ threshold of helium-like $\mathrm{Be}^{++}$ion are presented. The energies and the widths of the lowest ${ }^{1} S,{ }^{1} P,{ }^{1} D$, and ${ }^{1} \mathrm{~F}$ autoionizing states of the $\mathrm{Be}, \mathrm{Mg}$ atoms, and the lowest $\left({ }^{1} P\right)$ autoionizing states of $\mathrm{Ca}$ atom, are calculated. We consider briefly both a review of our previous results (together with results of other authors) and new calculations of our group. A brief review of the methods of the quasi-stationary states calculation is given.
\end{abstract}

Keywords Electron-impact Ionization of Atom, Autoionizing States, Quasi-stationary States, Interacting Configurations Method

\section{Introduction}

Investigations of autoionization phenomena in the framework of the problems dealing with the ionization and the electron scattering by atoms and ions were separated in the last decades into an independent branch of theoretical atomic physics. The scientific interest to the description of the processes of excitation and decay of quasi-stationary states is associated with a necessity to specify the parameters of elementary processes, which are used in theoretical estimations and calculations in plasma physics, laser spectroscopy, solid state physics, and crystallography, at the development of technological methods of isotope separation at the atomic le- vel, the designing of coherent ultra-violet and $\mathrm{x}$-ray radiation generators, as well as in other physical domains.

The results of experimental researches concerning the autoionizing states (AIS) located between the first and second ionization thresholds for helium and helium-like ions were qualitatively explained on the basis of the theory of isolated Fano resonance and in the diagonalization approximation. The appearance of new experimental data on resonance structures in partial cross-sections of helium photoionization above the threshold of excited ion formation (more exactly, in the interval between the second and third thresholds, to which the AIS energies converge in the atomic ionization problem) brought about a number of theoretical issues dealing, first of all, with the description of the interaction of a considerable number of overlapping quasi-stationary states, which decay through several open channels. Theoretical calculations and the analysis of resonance structures decaying into several states of a residual ion should be carried out, in the general case, with regard for all interconfiguration interactions.

One of the first theoretical methods that made it possible to obtain results coinciding with experimental data was the method of overlapping configurations or the method of interacting configurations. In the terminology adopted in this work, this formalism is called the method of interacting configurations in the real number representation (see Section 5). An important step of the theory became the method of interacting configurations in the complex number representation (ICCNR method). This method was developed in works [13 ] and successfully applied to the description of the quasistationary states of helium formed at its electron ionization in the energy interval above the threshold of excited ion formation.

At the modern stage in the development of this method, a principal possibility is its application to the calculation of ionization processes in more complicated atomic structures. Our way was a step by step transition from the He atom description [1-4], to the problem of ionization of $\mathrm{H}^{-}, \mathrm{Li}^{+}$ions 
$[5,6]$ and to the investigations of enough complex $\mathrm{Be}, \mathrm{Mg}$, $\mathrm{Ca}$ atoms, see, e. g., [7-13]. Few results in the photoionization problem on the ${ }^{1} P$ AIS above the $n=2$ threshold of helium-like $\mathrm{Be}^{++}$ion are presented as well. The results were reported at the international conferences [5, 7-10] as some approbation of the method and found data. The complete description of the method formalism was given in [4], see, e. g., [13] as well. In [14] the choice of the ground state wave function for such precision calculations of the quasistationary states parameters has been considered and discussed. The comparison with theoretical calculations in other methods has been considered as well.

As one can see in the literature, beryllium [15-36], magnesium $[15,16,18,37-51]$ and calcium [52-58] atoms turns out to be the promising objects for researches; $\mathrm{H}^{-}$[59-69], $\mathrm{Li}^{+}[70-76], \mathrm{Be}^{++}[71,72]$ ions, as well. In the start of our step by step program from helium to complex atoms we deal with the lowest AIS of $\mathrm{Be}, \mathrm{Mg}, \mathrm{Ca}$ atoms and $\mathrm{H}^{-}, \mathrm{Li}^{+}, \mathrm{Be}^{++}$ ions. Note that today the physics of resonances phenomena in atomic shells considers different quasi-stationary states, converging to the high-lying thresholds, see, e.g. [76, 77]. Moreover, different complicated processes in atomic shells are under consideration. Thus, the ICCNR method in future has the wide domain of applications.

\section{Few other well-defined and popu- lar methods of atomic characteris- tics calculations}

Since we deal with the quantum-mechanical three body problem (see the reaction (1) below) let us consider ((at least, briefly) the main methods for studying the three-particle quantum-mechanical scattering phenomena.

The Faddeev equations. The first well-defined mathematical method is based on Faddeev equations, see, e. g., [7880]. Indeed, some times the three body problem in quantum mechanics can be solved with the help of Faddeev equations. Nevertheless, this method is applied rarely for the calculations in atomic problems. The reason is in the long-range property of Coulomb potential and, as a consequence, in the slow convergence of the corresponding series. Therefore, usually the method is applied for the simplest atomic systems [7880]. As an example, the method was applied for the (e, $\mathrm{H})$ system, in which the hydrogen negative ion bound state and the lowest members of the resonances in both the singlet and the triplet $J=0$ series were calculated [78]. Further, in [79] the AIS of He atom were calculated. The resonance ionization by fast protons was studied, which was possible for the short-range nuclear potential. In [80] the scattering of electrons and positrons on hydrogen atom below the $n=2$ threshold was investigated. Thus, the Faddeev equations are not applied for the complex atoms AIS calculations. Therefore, the Faddeev-Merkuriev integral equations [81] look more preferable for the calculation of processes in atomic shell.

On the other hand both the Faddeev and ICCNR methods are based on the Lippmann-Schwinger-type equations. Indeed, the Faddeev formalism is started from the indication of the absence of uniqueness in the Lippmann-Schwinger solutions. This fact indicates some similarity between methods. However, the difference appears in T-operator analysis. Moreover, we do not appeal to three particle potential. Some other details about the three body problems with short-range interactions, application of Faddeev equations as well, can be found in [82].

The Fano method. Nevertheless, the start of theoretical calculations of resonance cross-sections in the photoionization problem is associated with the Fano paper [83]. The beginning of resonance profiles analysis can be found in [83] as well. The task of the new theory construction, which can take into account the coupled channels and can contain the spectroscopic characteristics of the interacting quasistationary states as the method parameters, has been formulated.In [84] the classification of two-electron excitation levels of helium has been suggested. Further, Fano demonstrates [85] that in the case of few interacting AIS, which decay into one open channel, the photoionization cross-section can be presented with explicit choosing of the members corresponding to the resonance process. The theory of isolated quasistationary state, which is observed in the cross-sections as the resonance, has been formulated by Fano in the paper [83]. In [86] the detailed description of the method has been given.

Diagonalization approximation. This method was very useful 50 years ago for investigations of the resonance scattering of slow electrons by many-electron atoms and ions. In this period it was a step forward. It was some unification of closed coupled channel approximation and interacting configuration method, in which only the diagonal elements of corresponding matrix were taken into account. The method was carried into atomic physics from the previous successful applications in the nuclear physics $[87,88]$. Thus, the diagonalization approximation was developed and realized by V.V. Balashov et al in articles [89, 90]. Further, see, e.g. [91], the method has been applied successfully for the quasi-stationary states description in the problems of photoionization and ionization of atoms by electron impact in the region between the first and second ionization thresholds. The form resonances have been described in this method in the paper [92]. The studied problems have a peculiarity. The excited quasistationary states decay occurs into one open channel. The detailed diagonalization method description can be found in [91]. The possibility of application to other type experiments has been considered. Moreover, the diagonalization approximation was very useful in the investigations of scattering of photons and electrons on complex atoms ions, see, e.g., V.I. Lengyel et al [93] and the references therein.

The Shore method. In main ideas and principles this method is near diagonalization method. It is shown that in the case of non-interacting resonances the total cross-section of 
ionization with few AIS excitation in the form of simple formulas can be found. The parameters look like the Fano formulae, see, e.g. [94]. Nevertheless, the author of the monograph [95] does not determine the explicit expressions for his parameters. Shore parameters are determined from the solution of the integral equation system with singular kerns. In the isolated resonance approximation the system of this linear parameters is equivalent to the Fano system of parameters. Today this system is widely used for the analysis and comparison of resonance curves, obtained in the experiments of different types.

Closed coupled channels approximation. The method was developed by Burke group in Belfast, see, e. g., [96, 97]. The important necessary principle has been realized. The formulation of this principle can be as follows. Theoretical calculations and the analysis of resonance structures decaying into several states of a residual ion should be carried out, in the general case, with taking into account the complete set of interconfiguration interactions $[96,97]$. Note that isolated resonance approximation does not lead to the coincidence with experimental data in such problems. The closed coupled channels approximation has been applied to the problems of photoionization and scattering of electrons by ions $[96,97]$. Further application of method was realized in wide-range problems of photon and electron collisions with atoms and ions. The details can be found in [98]. Quantummechanical close coupling approach to molecular collisions can be found in [99]. Thus, the closed coupled channel method [96-99] was a step forward in investigations of resonant processes.

Note on density functional theory. The difficulties in AIS theoretical description lead to the appearance of methods, which essentially depend from parameters taking from the experiment, not from the pure theory. Therefore, such approaches are not independent methods. The popular method of that kind is the density functional approach, see e.g. review in [100]. Thus, density functional theory, unfortunately, does not belong to so called exact quantum-mechanical methods. Moreover, the unique approach is absent. Many different variants of formalism have been developed by researches. Furthermore, in the problem of complex atoms AIS description all existing density functional approaches deal with essential difficulties. Therefore, obtained results are not of decisive character. Let us continue with independent theoretical approaches.

R-matrix method. A step forward in the development of the calculation technique in the framework of coordinate representation of the closed coupled channels approximation was given by R-matrix method, where the R-matrix formalism for the solution of the integro-differential equations system has been applied. Such method was suggested in the paper [101] of Wigner-Eisenbud for the solution of the nuclear physics problems. For the case of atomic physics the method has been modified in the papers of Burke, Taylor, Berringon, Eissner and Norrington, see, e.g. [102], where the calculation procedure has been presented in details. Today the R-matrix method is the most spread in the complex atoms AIS investigations $[36,102,103]$. This method is widely applied for the scattering of photons and electrons on the complex atoms both in ordinary [102] and B-spline [103] formalism.

The methods of interacting configurations. Here, contrary to the ICCNR method, this formalism is called the method of interacting configurations in the real number representation. It often becomes necessary to demonstrate the role of different multiconfiguration interactions in the resonance profiles formation, when the AIS excitation by the electrons and photons is studied. In the framework of coordinate representation of closed coupled channels method the realization of this procedure is difficult, because in the channel of reaction the separation of single configuration, which corresponds to the concrete quasi-stationary state excitation, is impossible (Note that AIS, which are different only in the main quantum number, belong to one and the same channel). The analysis of such problems is convenient to fulfill in the framework of the methods, which follows from the shell model of nucleus. The configuration interaction method, see, e.g. [104], has been used at first in the problems with configurations overlapping of many particle systems discrete spectrum in nucleus. In the papers of Feshbach [105] and Bloch [106] the direct generalization of the method for the states with continuous spectrum has been given. In atomic physics the problem of configuration interaction method equations application for the direct calculations of the distribution of the oscillations strengths transitions into the continuous spectrum has been considered by Fano [83-86]. Nevertheless, only the numerical methods of the integral equations solutions for the direct photoionization process have been formulated. In order to include the AIS calculation the method of [83-86] has been applied. Unfortunately, the obtaining of results for few AIS decay into few channels is impossible. This problem was overcome by the ICCNR method introducing, see, e.g. [1-4]. Note that the ordinary formalism of configuration interaction method (in our terminology the method of interacting configurations in real number representation) still is useful for calculations of processes in atomic shells [107].

The method of K-harmonics. This method has been described in details by Peterkop [108]. Some further development can be found in [109-111]. As it is ordinary for such methods the wave function expansion over some basis function is used. In order to simplify the method of solution the part of expansion, which is related to functions of the continuous spectrum and gives the integro-differential equations, is presented as the set in terms of K-harmonics or hyperspherical functions. Some exact formulae for K-harmonics method are given in [112].

The hyperspherical coordinates method. The method of K-harmonics is nearby to hyperspherical coordinates method, see, e.g., [66], in which the energetic positions of AIS can be calculated with good accuracy, but the calculation of widths meets some problems. This method has some simi- 
larity to Faddeev approach. The success of this method is evident, see, e.g., [66, 113]. However, the hyperspherical coordinates method is working good only for few body systems (here with Coulomb interaction too) and is not applied for the complex atoms AIS investigations as well.

The multiconfiguration Hartree-Fock method. Useful multiconfiguration Hartree-Fock method has been developed by C. Froese Fischer [114-116], especially the program for numerical calculations.

The random phase approximation with exchange method. The RPAE method is based on few physically available assumptions. The electrons in atom are considered as some dense gas. This assumption corresponds to low level of residual interaction of electrons in comparison with its kinetic energy. The method is described in details in [117]. The examples of application, e. g., for some states of Ar atom, are given in [118]. The application of multiconfiguration relativistic random-phase approximation for Be can be found in [19].

The method of complex coordinate rotation has been developed by Y. Ho [73, 119-121]. The essence of the method is the Ritz variational principle generalization, which gives the possibility for the calculations with complex test functions. At the manifold of the test function parameters the minimization of the Hamiltonians eigen values results as the vector of complex energies of the resonances under consideration. Note that method of complex coordinate rotation, as well as other methods bases on the Ritz variational principle, is formulated only for the calculations of wave functions, which describe the coupled states in two-electron systems, see, e. g., [73]. In [119] Hylleraas type wave functions are used to calculate resonance parameters for intrashell states (the two electrons occupy the same shell), and products of Slater-orbitals are used for intershell states (the two electrons occupy different shells). In [122] the configurationinteraction basis functions are applied.

\section{General description of the method}

In this section, the backgrounds of the method are briefly described.

The ICCNR method is a well-defined quantum-mechanical method for the calculation of parameters of atomic systems. This method is a development and a generalization of the known method of interacting configurations in the real number representation. It has some advantages in comparison with the standard method of interacting configurations in the real number representation and other calculation methods for the energies and widths of quasi-stationary atomic states. First, this is a capability of finding not only the energies, but also the widths of quasi-stationary states. Second, there are new possibilities for the resonance identification. The ICCNR method makes it possible, on the basis of the results of calculations, to estimate the contribution of each resonance state to the cross-section of the process and, if the resonance approximation is applicable, to introduce a set of parameters that determine the energies and the widths of quasi-stationary states, as well as the contours of resonance lines in the ionization cross-sections. This approach also enables the applicability of approximate methods to the estimation of crosssections in specific problems to be studied and the limits of their validity to be determined. Those advantages make it possible to successfully apply the ICCNR method not only to scattering processes, but also to much more complicated processes of atom ionization by electrons.

\section{Motivation and goals}

We presented here our step by step transition from the $\mathrm{He}$ atom description via the problem of ionization of $\mathrm{H}^{-}, \mathrm{Li}^{+}$ ions up to the enough complex atoms (such as $\mathrm{Be}, \mathrm{Mg}$ and $\mathrm{Ca}$ ) investigations. One of the goals of these investigations is to demonstrate that the ICCNR method can be useful for the complex atoms study on the level of popular R-matrix approach, see, e. g., [36].

The ICCNR method is applied here to the calculation of spectroscopic characteristics of AIS of $\mathrm{Be}, \mathrm{Mg}$ and $\mathrm{Ca}$ atoms in the problem of the electron-impact ionization of these atoms. In particular, the energies and the widths of the lowest $\left({ }^{1} S,{ }^{1} P,{ }^{1} D\right.$, and $\left.{ }^{1} F\right)$ AIS of Be, $\mathrm{Mg}$ and Ca atoms are calculated. The important stage is the problem of ionization of $\mathrm{H}^{-}, \mathrm{Li}^{+}$ions.

The exact quantum-mechanical methods are welcome here because the experimental investigations of beryllium are complicated due to its chemical properties. Furthermore, the atomic shell of Be atom is not simple and is not very complex. Therefore, this atom is enough suitable object for the beginning of the application of the ICCNR method to the compound atomic systems investigations.

Here some results for Be atom [15-20] are compared with the calculations on the basis of ICCNR method. Further analysis of literature shows that beryllium atom until today is the interesting experimental and theoretical problem [15-36]. The authors of [15-36] prefer the approbation of their experimental and theoretical methods in the investigations of processes in the beryllium atomic shell.

Similarly, some results for $\mathrm{Mg}$ atom [18, 37, 38] are compared with our calculations in ICCNR method. Furthermore, our results (found in ICCNR method) for Ca atom are compered with experimental and theoretical investigations [52-54].

One of the goals of our research is to illustrate the capabilities of the ICCNR method in the determination of spectroscopic characteristics of complicated atoms. Quasi-stationary states were studied in such multielectron atomic systems as $\mathrm{Be}, \mathrm{Mg}, \mathrm{Ca}$ atoms and $\mathrm{H}^{-}, \mathrm{Li}^{+}$ions [5, 7-10]. The capabilities of the method were illustrated briefly by the example of the atomic ionization by the electron impact [5, 7-10], which are challenging for researches. The analysis of the loss spectrum of ejected electrons made it possible to compare indirectly the obtained results with the results of studies of the 
scattering problem. The results were reported at the international conferences [5, 7-10]. This journal presentation is the expanded consideration of [5, 7-10].

\section{Formalism of the method}

Let us recall the foundations of the ICCNR method for the study of the processes of atomic ionization by the electron impact. Consider the equation of the examined reaction

$$
A\left(n_{0} L_{0} S_{0}\right)+e^{-}\left(\vec{k}_{0}\right) \rightarrow A^{+}\left(n l_{1}\right)+e^{-}\left(\vec{k}_{1}\right)+e^{-}(\vec{k}),
$$

where $\vec{k}_{0}, \vec{k}_{1}, \vec{k}$ are the momenta of the incident, ejected, and scattered electrons, respectively. Then the generalized oscillator strength of the transition for the incident electron in the Born approximation is given by

$$
\frac{d f_{n l_{1}}}{d E}(Q)=\frac{E}{Q^{2}} \sum_{l L}\left|\left\langle n L_{1} E l\left|\sum_{j=1}^{n} \exp \left(i \vec{Q} \vec{r}_{i}\right)\right| n_{0} L_{0} S_{0}\right\rangle\right|^{2}
$$

In this formula $E=k_{0}^{2}-k^{2}$ is the energy loss, $\vec{Q}=\overrightarrow{k_{0}}-$ $\vec{k}$ is the transmitted momentum, and $\left|n l_{1} E l: L S_{0}\right\rangle$ is the wave function of an atom with total momentum $L$ and spin $S_{0}$ provided that an electron with momentum $l$ and energy $E$ is in the field of ion $A^{+}$, whose electron has the quantum numbers $\left|n l_{1}\right\rangle$. The function of the atomic ground state is given by $\left|n_{0} L_{0} S_{0}\right\rangle$.

Note that process (1) is a much more complicated physical phenomenon in comparison with the electron scattering by an atom. Exact theoretical calculations of such processes constitute a problem for modern theoretical physics. Therefore, the consideration of this problem for multielectron atoms in the framework of the ICCNR method is an important and challenging scientific step.

The choice of the wave function for the ground state is dictated by a desirable accuracy of the final results of calculations. In the case of two-electron systems, this is a multiparametric Hylleraas-type wave function [123], and, in the case of $\mathrm{Be}$ atom, this is, as a rule, a Hartree-Fock wave function obtained in the multiconfiguration approximation [114-116]. In our papers we used the Tweed [124] wave function as well, see [14] for details. The system of equations in the ICCNR method has the following form:

$$
\begin{gathered}
\left(E_{n}-E\right) a_{\lambda n}^{E i}+\sum_{\lambda^{\prime}} \int_{0}^{\infty} b_{\lambda \lambda^{\prime}}^{E i}\left(E^{\prime}\right) V_{n \lambda^{\prime}}\left(E^{\prime}\right) d E^{\prime} \\
\sum_{m} a_{\lambda m}^{E i} V_{m \lambda^{\prime}}^{*}\left(E^{\prime}\right)+\left(E^{\prime}-E\right) b_{\lambda \lambda^{\prime}}^{E i}\left(E^{\prime}\right)=0 .
\end{gathered}
$$

The multipliers $a_{\lambda m}^{E i}$ and $b_{\lambda \lambda^{\prime}}^{E i}\left(E^{\prime}\right)$ are the coefficients of expansion of the wave function $\Psi_{\lambda}^{E}\left(\overrightarrow{r_{1}}, \overrightarrow{r_{2}}\right)$ in the basis

$$
\Psi_{\lambda}^{E}\left(\overrightarrow{r_{1}}, \overrightarrow{r_{2}}\right)=\sum_{m} a_{\lambda m}^{E i}|m\rangle+\sum_{\lambda^{\prime}} \int_{0}^{\infty} b_{\lambda \lambda^{\prime}}^{E i}\left(E^{\prime}\right)\left|\lambda^{\prime} E^{\prime}\right\rangle d E^{\prime}
$$

The basis wave functions satisfy the conditions

$$
\langle m|\widehat{H}| n\rangle=E_{n} \delta_{n m},\left\langle\lambda^{\prime} E^{\prime}|\widehat{H}| \lambda E\right\rangle=E \delta_{\lambda \lambda^{\prime}} \delta\left(E-E^{\prime}\right),
$$

where $\widehat{H}$ is the total Hamiltonian of the system.

The formal solution for the multiplier $b_{\lambda \lambda^{\prime}}^{E i}\left(E^{\prime}\right)$ is given by

$$
\begin{gathered}
b_{\lambda \lambda^{\prime}}^{E i}\left(E^{\prime}\right)=P \frac{\sum_{m} a_{\lambda m}^{E i} V_{m \lambda}(E)}{E-E^{\prime}}+\left[A_{\lambda \lambda^{\prime}}\right. \\
\left. \pm i \pi \sum_{m} a_{\lambda m}^{E i} V_{m \lambda^{\prime}}(E)\right] \delta\left(E-E^{\prime}\right),
\end{gathered}
$$

where $V_{m \lambda}(E)=\langle m|\widehat{H}| \lambda E\rangle$. The matrix $A_{\lambda \lambda^{\prime}}$ depends on the asymptotic properties of the basis functions $|\lambda E\rangle$. Substitution of Eq. (6) into Eq. (3) transforms the system of equations obtained in the ICCNR method into a system of linear algebraic equations for the coefficients $a_{\lambda m}^{E i}$ :

$$
\begin{gathered}
\left(E_{n}-E\right) a_{\lambda n}^{E i}+\sum_{m}\left[F_{n m}(E)-i \gamma_{n m}(E)\right] a_{\lambda m}^{E i} \\
=-\sum_{\lambda^{\prime}} A_{\lambda \lambda^{\prime}} V_{\lambda^{\prime} n}(E)
\end{gathered}
$$

The latter can be expressed in terms of eigenvectors and eigenvalues of the complex matrix

$$
W_{n m}(E)=E_{n} \delta_{n m}+F_{n m}(E)-i \gamma_{n m}(E),
$$

where

$$
\begin{gathered}
\gamma_{n m}(E)=\pi \sum_{\lambda} V_{n \lambda}(E) V_{\lambda m}(E) ; \\
F_{n m}(E)=\frac{1}{\pi} \int_{0}^{\infty} \frac{\gamma_{n m}(E)}{E-E^{\prime}} d E^{\prime} .
\end{gathered}
$$

The analysis of formulas (8) and (9) allows one to compare various approximations, which can be done in the ICCNR method. One can see that, in the framework of this method, the following approximations are possible:

1) the method of interacting configurations in the real number representation; this approximation corresponds to the neglect of complex components $i \gamma_{n m}(E)$ in matrix (8);

2) the diagonalization approximation in the real number representation consists in that the sum of all non-diagonal members $F_{n m}(E)-i \gamma_{n m}(E)$ in the matrix $W_{n m}(E)$ is neglected;

3) the diagonalization approximation involving the transitions outside the energy surface (or the diagonalization approximation in the complex number representation) arises if the term $F_{n m}(E)$ is neglected in calculations.

The account for all members in matrix (8) is, in essence, the ICCNR method, the advantages of which over the indicated approximations are obvious.

After determining the eigenvectors and eigenvalues of the matrix $W_{n m}(E)$, we can calculate the energies and widths of quasi stationary states that are located above the threshold of 
excited ion formation [1, 2]. The partial amplitudes of the resonance ionization can be determined as follows:

$$
T_{|0\rangle \rightarrow|\lambda E\rangle}(E)=t_{\lambda}^{d i r}(E)+\sum_{m} \frac{H_{m \lambda(E)}}{\varepsilon_{m}(E)+1} .
$$

The quantities in formula (10) are defined by the relations

$$
\begin{gathered}
t_{\lambda}^{d i r}(E)=\sqrt{C(E)}\langle\lambda E|\hat{t}| 0\rangle, \\
H_{m \lambda}(E)=2 \widetilde{V}_{m \lambda}(E)\left[t_{m}(E)-i \tau_{m}(E)\right] \Gamma_{m}^{-1}(E),
\end{gathered}
$$

where

$$
t_{m}(E)=\sqrt{C(E)}\left\langle\widetilde{F}_{m}^{E}|\hat{t}| 0\right\rangle, \quad \tau_{m}(E)=\sqrt{C(E)}\left\langle\chi_{m}^{E}|\hat{t}| 0\right\rangle .
$$

Hence, the expressions for the cross-sections become parametrized

$\sigma_{\lambda}(E)=\sigma_{\lambda}^{d i r}(E)+\sum_{m} \frac{\Gamma_{m}(E) P_{m \lambda}(E)+\varepsilon_{m}(E) Q_{m \lambda}(E)}{\varepsilon_{m}^{2}(E)+1}$.

The real functions $P_{m \lambda}(E)$ and $Q_{m \lambda}(E)$ of the total energy $E$ are the doubled real and imaginary, respectively, parts of the complex function $N_{m \lambda}(E)$, which looks like

$N_{\alpha m}(E)=\sum_{\lambda \varepsilon \alpha} H_{m \lambda}(E)\left(t_{\lambda}^{d i r}(E)+\sum_{n} \frac{H_{m \lambda}(E)}{\varepsilon_{n}(E)-\varepsilon_{m}(E)+2 i}\right)^{*}$.

Hence, the resonance ionization cross-section is determined by a collection of the following functions of the total energy $E: \sigma_{\lambda}^{d i r}(E), N_{\alpha m}(E), \varepsilon_{m}(E)$, and $\Gamma_{m}(E)$. See more details about the formalism of the method (for two electron systems) in the article [4].

\section{The results of the calculations}

Here the electron-impact ionization of the $\mathrm{H}^{-}, \mathrm{Li}^{+}$ions and $\mathrm{Be}, \mathrm{Mg}, \mathrm{Ca}$ atoms in the interval of AIS excitation are considered.

\subsection{The positions of the autoionizing states of the $\mathrm{H}^{-}$ and $\mathrm{Li}^{+}$ions, converging to the threshold $\mathrm{n}=3$}

The results of the calculations for electron-impact ionization of $\mathrm{H}^{-}, \mathrm{Li}^{+}$ions are presented. The ICCNR method is used. The positions of four lowest AIS of the $\mathrm{H}^{-}$ion and three lowest $\mathrm{Li}^{+}$ions, converging to the threshold $\mathrm{n}=3$, are given in the Tables 1 and 2, respectively. The comparison with experimental and theoretical results of other authors is presented in these tables as well.

Furthermore, first and forth resonances of Table 1 can be compared with AIS $\mathrm{E}=12.650, \Gamma=0.02758$ and $\mathrm{E}=12.837$, $\Gamma=0.00163$ in [59], respectively. Moreover, three quasistationary states of Table 2 can be compared with resonances $\mathrm{E}=175.80, \mathrm{E}=178.18$ and $\mathrm{E}=179.37$ in [70], respectively.
Table 1. Energies and widths of the lowest AIS of the $\mathrm{H}^{-}$ion, converging to the threashold $\mathrm{n}=3$, in the ICCNR method

\begin{tabular}{|c|c|c|c|c|}
\hline No & $\mathrm{E}, \mathrm{eV}$ & $\Gamma, \mathrm{eV}$ & $\mathrm{E}, \mathrm{eV}[63]$ & $\Gamma, \mathrm{eV}[63]$ \\
\hline 1 & 12.6598 & 0.0304 & 12.6586 & 0.0329 \\
\hline 2 & 12.7801 & 0.0010 & 12.7677 & 0.0012 \\
\hline 3 & 12.8479 & 0.0026 & 12.8382 & 0.0030 \\
\hline 4 & 12.8591 & 0.0018 & 12.8416 & 0.0022 \\
\hline No & $\mathrm{E}, \mathrm{eV}$ & $\Gamma, \mathrm{eV}$ & $\mathrm{E}, \mathrm{eV}[60]$ & $\mathrm{E}, \mathrm{eV}[61]$ \\
\hline 1 & 12.6598 & 0.0304 & 12.6605 & 12.6602 \\
\hline 2 & 12.7801 & 0.0010 & 12.7656 & 12.7658 \\
\hline 3 & 12.8479 & 0.0026 & 12.8330 & 12.8332 \\
\hline 4 & 12.8591 & 0.0018 & 12.8394 & 12.8408 \\
\hline
\end{tabular}

Table 2. Energies and widths of the lowest ${ }^{1} P$ AIS of the $\mathrm{Li}^{+}$ion, converging to the threshold $n=3$, in the ICCNR method

\begin{tabular}{|c|c|c|c|c|}
\hline${ }^{1} P$ & $\mathrm{E}, \mathrm{eV}$ & $\Gamma, \mathrm{eV}$ & $\mathrm{E}, \mathrm{eV}[72]$ & $\Gamma, \mathrm{eV}[72]$ \\
\hline 1 & 175.58 & 0,281 & 175.77 & 0.321 \\
\hline 2 & 178.27 & 0,071 & 178.58 & 0.078 \\
\hline 3 & 179.45 & 0,016 & 179.60 & 0.019 \\
\hline${ }^{1} P$ & $\mathrm{E}, \mathrm{eV}$ & $\Gamma, \mathrm{eV}$ & $\mathrm{E}, \mathrm{eV}[71]$ & $\Gamma, \mathrm{eV}[71]$ \\
\hline 1 & 175.58 & 0,281 & 175.49 & 0.272 \\
\hline 2 & 178.27 & 0,071 & 178.10 & 0.068 \\
\hline 3 & 179.45 & 0,016 & - & - \\
\hline
\end{tabular}

\subsection{Energies and widths of the lowest autoionizing sta- tes of Be atom}

In brief report [7], using the ICCNR method, the investigation of the electron-impact ionization of a Be atom in the AIS excitation interval has been started, and the spectra of energy loss were analyzed. The photoionization of this atom has been studied as well. The AIS that arise in this problem can be compared with the AIS that are formed in the problem of electron scattering at the corresponding ion. In calculations, the Coulomb wave functions were used as basis configurations. For every term, up to 25 basis configurations were taken into account.

Table 3 contains the results of our calculations for the energies and the widths of the lowest AIS of a Be atom $\left({ }^{1} S,{ }^{1} P,{ }^{1} D\right.$, and $\left.{ }^{1} F\right)$. These resonances are found in the ICCNR approximation in the problem of the electron-impact ionization of an atom. These results are compared with the energies and the widths of AIS obtained in the paper [18], where another problem of electron scattering by a $\mathrm{Be}^{+}$ion is discussed. Therefore, such comparison is indirect. Furthermore, in Table 4, the energies of ${ }^{1} P$ states, which are located between the first and second ionization thresholds of a beryllium atom, are compared with the results of calculations obtained by other authors [15-20]. 
Table 3. Energies and widths of the lowest AIS $\left({ }^{1} S,{ }^{1} P,{ }^{1} D\right.$, and $\left.{ }^{1} F\right)$ of a beryllium atom obtained in the ICCNR approximation in the problem of the electron-impact ionization of an atom. In the paper [18], the energies of AIS were calculated in the diagonalization approximation in the framework of the problem of electron scattering by a $\mathrm{Be}^{+}$ion

\begin{tabular}{|c|c|c|c|c|}
\hline${ }^{1} S$ & $\mathrm{E}, \mathrm{eV}$ & $\Gamma, \mathrm{eV}$ & $\mathrm{E}, \mathrm{eV}[18]$ & $\Gamma \mathrm{eV}[18]$ \\
\hline $3 s^{2}$ & 16.42 & 0.0803 & 16.40 & 0.0818 \\
\hline $3 p^{2}$ & 18.65 & 0.0110 & 18.57 & 0.0116 \\
\hline $3 s 4 s$ & 18.82 & 0.0351 & 18.74 & 0.0358 \\
\hline $3 s 5 s$ & 19.48 & 0.0163 & 19.45 & 0.0167 \\
\hline $3 s 6 s$ & 19.77 & 0.00869 & 19.75 & 0.00884 \\
\hline $3 s 7 s$ & 19.96 & 0.00518 & 19.92 & 0.00527 \\
\hline${ }^{1} P$ & $\mathrm{E}, \mathrm{eV}$ & $\Gamma \mathrm{eV}$ & $\mathrm{E}, \mathrm{eV}[18]$ & $\Gamma \mathrm{eV}[18]$ \\
\hline $3 s 3 p$ & 17.70 & 0.157 & 17.68 & 0.169 \\
\hline $3 s 4 p$ & 18.85 & 0.0318 & 18.83 & 0.0321 \\
\hline $3 s 5 p$ & 19.45 & 0.00601 & 19.41 & 0.0062 \\
\hline $3 s 6 p$ & 19.73 & 0.0157 & 19.68 & 0.0161 \\
\hline $3 p 4 s$ & 19.81 & 0.00328 & 19.77 & 0.0033 \\
\hline $3 s 7 p$ & 19.89 & 0.0274 & 19.82 & 0.0282 \\
\hline $3 s 8 p$ & 19.95 & 0.0140 & 19.93 & 0.0143 \\
\hline${ }^{1} D$ & $\mathrm{E}, \mathrm{eV}$ & $\Gamma \mathrm{eV}$ & $\mathrm{E}, \mathrm{eV}[18]$ & $\Gamma \mathrm{eV}[18]$ \\
\hline $3 s 3 d$ & 17.62 & 0.0214 & 17.56 & 0.0220 \\
\hline $3 p^{2}$ & 18.31 & 0.0224 & 18.67 & 0.0230 \\
\hline $3 s 4 d$ & 19.09 & 0.0378 & 19.09 & 0.0389 \\
\hline $3 s 5 d$ & 19.60 & 0.0121 & 19.56 & 0.0128 \\
\hline $3 d^{2}$ & 19.67 & 0.00789 & 19.63 & 0.0796 \\
\hline $3 s 6 d$ & 19.81 & 0.00331 & 19.79 & 0.0034 \\
\hline${ }^{1} F$ & $\mathrm{E}, \mathrm{eV}$ & $\Gamma \mathrm{eV}$ & $\mathrm{E}, \mathrm{eV}[18]$ & $\Gamma \mathrm{eV}[18]$ \\
\hline $3 p 3 d$ & 18.96 & 0.0203 & 18.95 & 0.0214 \\
\hline $3 s 4 f$ & 19.43 & 0.0149 & 19.43 & 0.0155 \\
\hline $3 s 5 f$ & 19.72 & 0.0070 & 19.70 & 0.00717 \\
\hline $3 s 6 f$ & 19.88 & 0.0023 & 19.85 & 0.00235 \\
\hline $3 s 7 f$ & 19.95 & 0.00021 & 19.94 & 0.00023 \\
\hline $3 s 8 f$ & 19.97 & 0.0019 & - & - \\
\hline & & & & \\
\hline
\end{tabular}

In the literature, there are no similar results obtained on the basis of exact computational methods, in particular, on the basis of the method of interacting configurations and, moreover, on the basis of the ICCNR one. The comparison with the results of paper [18] found in the diagonalization approximation (see Table 3 ) is indirect, because it deals with a different object in a different problem. Nevertheless, it really evidences the reliability of the results obtained here.

Here we are able to add energies and the widths in the photoionization problem of the ${ }^{1} P$ AIS below the $n=3$ threshold of helium-like $\mathrm{Be}^{++}$ion. The first three ${ }^{1} P$ resonances above the $n=2$ threshold are presented. The results are compared with theoretical calculations of [78, 79]. These results are presented in Table 5.
Table 4. Comparison of the energies obtained with the use of the ICCNR method for the AIS of a beryllium atom, which are located between the corresponding first and second ionization thresholds, with the results of other authors

\begin{tabular}{|c|c|c|c|c|}
\hline${ }^{1} P$ & $\mathrm{E}, \mathrm{eV}$ & $\mathrm{E}, \mathrm{eV}[15]$ & $\mathrm{E}, \mathrm{eV}[16]$ & $\mathrm{E}, \mathrm{eV}[17]$ \\
\hline $2 p 3 s$ & 10.71 & 10.71 & 10.93 & 10.77 \\
\hline $2 p 3 d$ & 10.84 & 11.86 & 11.86 & 11.86 \\
\hline $2 p 4 s$ & 12.03 & 11.97 & 12.10 & 12.07 \\
\hline $2 p 4 d$ & 12.42 & 12.47 & 12.50 & 12.49 \\
\hline${ }^{1} P$ & $\mathrm{E}, \mathrm{eV}$ & $\mathrm{E}, \mathrm{eV}[18]$ & $\mathrm{E}, \mathrm{eV}[19]$ & $\mathrm{E}, \mathrm{eV}[20]$ \\
\hline $2 p 3 s$ & 10.71 & 10.73 & 10.63 & 10.91 \\
\hline $2 p 3 d$ & 10.84 & 11.85 & 12.03 & 11.83 \\
\hline $2 p 4 s$ & 12.03 & 12.09 & 12.09 & 12.09 \\
\hline $2 p 4 d$ & 12.42 & 12.49 & 12.61 & 12.44 \\
\hline
\end{tabular}

Table 5. Comparison of the energies and the widths obtained with the use of the ICCNR method for the AIS below the $n=3$ threshold of a helium-like $\mathrm{Be}^{++}$ion with the theoretical results of other authors (the first three ${ }^{1} P$ resonances above the $\mathrm{n}=2$ threshold are under consideration)

\begin{tabular}{|c|c|c|c|c|c|}
\hline $\mathrm{E}, \mathrm{eV}$ & $\Gamma, \mathrm{eV}$ & $\mathrm{E}, \mathrm{eV}[71]$ & $\Gamma, \mathrm{eV}[71]$ & $\mathrm{E}, \mathrm{eV}[72]$ & $\Gamma, \mathrm{eV}[72]$ \\
\hline 329.18 & 0.318 & 329.50 & 0.324 & 329.55 & 0.412 \\
\hline 333.24 & 0.0081 & 333.35 & 0.086 & 333.69 & 0.088 \\
\hline 337.47 & 0.0019 & - & - & 337.66 & 0.0023 \\
\hline
\end{tabular}

\subsection{Electron-impact ionization of a $\mathrm{Mg}$ atom in the in- terval of the excitation of autoionizing states}

The investigation of the ionization of $\mathrm{Mg}$ atoms (and $\mathrm{Mg}^{+}$ ions) by photons and electrons is a challenging problem, which is proved by both experimental and theoretical papers of many authors (see, e.g., publications [8, 9, 18, 37, 38] considered here). In brief articles [8, 9], we started to study the electron-impact ionization of a $\mathrm{Mg}$ atom in the AIS excitation interval with the use of the ICCNR method. In Table 6, the results of our calculations for the energies and the widths of the lowest AIS $\left({ }^{1} S,{ }^{1} P,{ }^{1} D\right.$, and $\left.{ }^{1} F\right)$ of a $\mathrm{Mg}$ atom obtained in the electron-impact ionization problem in the ICCNR approximation are presented.

First, our results are compared with similar states that are formed in the problem of electron scattering by $\mathrm{Mg}^{+}$ions [18] (see Table 6). Since another problem has been considered in paper [18] - namely, the scattering one - such a comparison is indirect. In [18] the calculations were carried out in the diagonalization approximation. Second, in the framework of the problem of the electron-impact ionization of atoms, the energies of ${ }^{1} P$-states must coincide with those obtained in the problem of photoionization of a $\mathrm{Mg}$ atom. Therefore, a direct comparison of our results with experimental ones [37] and with the results of calculations on the basis 
of the R-matrix method [38] can be made. In Table 7, the energy positions and the widths calculated for the ${ }^{1} P$ AIS of a magnesium atom with the use of the ICCNR method are directly compared with the experimental data of paper [37] and the theoretical data obtained with the help of the R-matrix formalism [38], as well as with the problem of electron scattering by a $\mathrm{Mg}^{+}$ion [18].

Table 6. Energies and widths of the lowest AIS $\left({ }^{1} S,{ }^{1} P,{ }^{1} D\right.$, and $\left.{ }^{1} F\right)$ of a $\mathrm{Mg}$ atom obtained in the ICCNR approximation in the problem of electronimpact ionization of an atom. In paper [18] the energies of autoionizing states were calculated in the diagonalization approximation in the framework of the problem of electron scattering by a $\mathrm{Mg}^{+}$ion

\begin{tabular}{|c|c|c|c|c|}
\hline${ }^{1} S$ & $\mathrm{E}, \mathrm{eV}$ & $\Gamma \mathrm{eV}$ & E, eV [18] & $\Gamma \mathrm{eV}[18]$ \\
\hline $4 s^{2}$ & 13.08 & 0.0987 & 13.06 & 0.1010 \\
\hline $3 d^{2}$ & 14.61 & 0.0480 & 14.66 & 0.0502 \\
\hline $4 s 5 s$ & 14.92 & 0.0425 & 14.97 & 0.0473 \\
\hline $4 s 6 s$ & 15.48 & 0.0196 & 15.53 & 0.0185 \\
\hline$\overline{3 d 4 d}$ & 15.59 & 0.0140 & 15.64 & 0.0129 \\
\hline $4 s 7 s$ & 15.78 & 0.0115 & 15.80 & 0.0107 \\
\hline $4 s 8 s$ & 15.80 & 0.0069 & - & - \\
\hline${ }^{1} P$ & $\mathrm{E}, \mathrm{eV}$ & $\Gamma \mathrm{eV}$ & $\mathrm{E}, \mathrm{eV}[18]$ & $\Gamma \mathrm{eV}[18]$ \\
\hline $4 s 4 p$ & 14.15 & 0.157 & 14.18 & 0.143 \\
\hline $3 d 4 p$ & 15.01 & 0.172 & 14.95 & 0.162 \\
\hline $4 s 5 p$ & 15.34 & 0.0324 & 15.29 & 0.0301 \\
\hline $4 s 6 p$ & 15.68 & 0.0682 & 15.64 & 0.0667 \\
\hline $3 d 4 f$ & 15.77 & 0.0481 & 15.74 & 0.0448 \\
\hline $4 s 7 p$ & 15.85 & 0.0059 & 15.86 & 0.0048 \\
\hline $3 s 8 p$ & 19.95 & 0.0140 & 19.93 & 0.0143 \\
\hline${ }^{1} D$ & $\mathrm{E}, \mathrm{eV}$ & $\Gamma \mathrm{eV}$ & $\mathrm{E}, \mathrm{eV}[18]$ & $\Gamma \mathrm{eV}[18]$ \\
\hline $3 d 4 s$ & 13.62 & 0.262 & 13.66 & 0.272 \\
\hline $3 d^{2}$ & 14.31 & 0.253 & 14.38 & 0.269 \\
\hline $4 d 4 s$ & 14.89 & 0.0192 & 14.96 & 0.0189 \\
\hline $3 d 5 s$ & 15.28 & 0.0869 & 15.30 & 0.0951 \\
\hline $4 p^{2}$ & 15.47 & 0.0570 & 15.49 & 0.0578 \\
\hline $3 d 4 d$ & 15.58 & 0.0865 & 15.55 & 0.0876 \\
\hline $4 s 5 d$ & 15.69 & 0.0258 & 15.66 & 0.0248 \\
\hline${ }^{1} F$ & $\mathrm{E}, \mathrm{eV}$ & $\Gamma \mathrm{eV}$ & $\mathrm{E}, \mathrm{eV}[18]$ & $\Gamma \mathrm{eV}[18]$ \\
\hline $3 d 4 p$ & 14.15 & 0.0225 & 14.66 & 0.0230 \\
\hline $4 s 4 f$ & 15.01 & 0.0110 & 15.28 & 0.0113 \\
\hline $3 d 5 p$ & 15.34 & 0.0540 & 15.53 & 0.0589 \\
\hline $3 d 4 f$ & 15.53 & 0.0052 & 15.63 & 0.0053 \\
\hline $4 s 5 f$ & 15.68 & 0.0201 & 15.71 & 0.0205 \\
\hline $3 d 6 p$ & 15.77 & 0.0104 & 15.88 & 0.0109 \\
\hline $4 s 6 f$ & 15.85 & 0.0125 & 15.90 & 0.0131 \\
\hline
\end{tabular}

Thus, the original scientific results found with the help of the ICCNR method for the energies and the widths of the lowest AIS $\left({ }^{1} S,{ }^{1} P,{ }^{1} D\right.$, and $\left.{ }^{1} F\right)$ of a $\mathrm{Mg}$ atom in the problem of electron-impact ionization of this atom are presented (see Table 6). Their novelty consists in the application of the exact calculation method, namely, the method of interacting confi- gurations and, the more so, the ICCNR method. The comparison with the calculations of corresponding energies and widths of AIS carried out in the diagonalization approximation in the problem of electron scattering by $\mathrm{Mg}^{+}$ions (Table 6 ) is indirect (a different object in a different problem), but really testifies to the reliability of the results obtained. Some of the results obtained here, namely, the energy positions of the ${ }^{1} \mathrm{P}$ AIS of a Mg atom, can be directly compared with the experiment and the R-matrix calculations (see Table 7). The results of calculations carried out with the use of the ICCNR method are in good agreement with the corresponding calculations using the R-matrix method [38] and experimental results [37] (see Table 7).

Table 7. Comparison of the energies and the widths of the AIS of a magnesium atom obtained with the use of the ICCNR method with the experiment [37] and R-matrix calculations for ${ }^{1} P$-states [38] (paper [38]: the photoionization problem and the photoionization threshold; work [18]: the scattering problem)

\begin{tabular}{|c|c|c|c|c|c|}
\hline${ }^{1} P$ & $\mathrm{E}, \mathrm{eV}$ & $\Gamma \mathrm{eV}$ & E, eV [18] & $\Gamma \mathrm{eV}[18]$ & \\
\hline $4 s 4 p$ & 14.15 & 0.157 & 14.18 & 0.143 & \\
\hline $3 d 4 p$ & 15.01 & 0.172 & 14.95 & 0.162 & \\
\hline $4 s 5 p$ & 15.34 & 0.0324 & 15.29 & 0.0301 & \\
\hline $3 d 5 p$ & 15.53 & 0.0775 & 15.56 & 0.0758 & \\
\hline $4 s 6 p$ & 15.68 & 0.00682 & 15.64 & 0.00667 & \\
\hline $3 d 4 f$ & 15.77 & 0.0481 & 15.74 & 0.0448 & \\
\hline $4 s 7 p$ & 15.85 & 0.00592 & 15.86 & 0.00476 & \\
\hline $4 s 8 p$ & 15.90 & 0.0087 & - & - & \\
\hline $3 d 6 p$ & 15.93 & 0.0295 & - & - & \\
\hline $4 s 9 p$ & 15.95 & 0.0011 & - & - & \\
\hline${ }^{1} P$ & $\mathrm{E}, \mathrm{eV}$ & $\Gamma \mathrm{eV}$ & E, eV [38] & $\Gamma \mathrm{eV}[38]$ & $\mathrm{E}, \mathrm{eV}$ [37] \\
\hline $4 s 4 p$ & 14.15 & 0.157 & 14.2213 & 0.3921 & 14.18 \\
\hline $3 d 4 p$ & 15.01 & 0.172 & 14.9048 & 0.6078 & - \\
\hline $4 s 5 p$ & 15.34 & 0.0324 & 15.3133 & 0.0931 & - \\
\hline $3 d 5 p$ & 15.53 & 0.0775 & 15.7264 & 0.0890 & 15.24 \\
\hline $4 s 6 p$ & 15.68 & 0.00682 & 15.6653 & 0.0142 & 15.61 \\
\hline $3 d 4 f$ & 15.77 & 0.0481 & - & - & - \\
\hline $4 s 7 p$ & 15.85 & 0.00592 & 15.8675 & 0.0095 & 15.83 \\
\hline $4 s 8 p$ & 15.90 & 0.0087 & 15.9802 & 0.0111 & 15.98 \\
\hline $3 d 6 p$ & 15.93 & 0.0295 & 16.007 & 0.0417 & - \\
\hline $4 s 9 p$ & 15.95 & 0.0011 & 16.065 & 0.0019 & 16.06 \\
\hline
\end{tabular}

\subsection{Electron-impact ionization of a Ca atom in the in- terval of the excitation of autoionizing states}

The application of ICCNR method to calculate the lowest AIS of calcium atom was begun in work [10]. The energies and the widths of the lowest ${ }^{1} P$-states were calculated. The results were compared with the data obtained by other authors. In Table 8, besides the results of our calculations [10], the experimental data [52] and the results of theoretical calculations $[53,54]$ are shown. Their analysis testifies that the classification of AIS proposed in work [53] is possible. The results of our calculations agree well with the theoretical data 
obtained by other authors.

Table 8. Comparison of the energies and the widths obtained with the use of the ICCNR method for the AIS of a Ca atom with the theoretical results of other authors [53, 54] and the experiment [52]

\begin{tabular}{|c|c|c|c|c|}
\hline${ }^{1} P$ & $\mathrm{E}, \mathrm{eV}$ & $\mathrm{E}, \mathrm{eV}[52]$ & $\mathrm{E}, \mathrm{eV}[53]$ & $\mathrm{E}, \mathrm{eV}[54]$ \\
\hline $3 d 5 p$ & 6.601 & 6.59 & 6.604 & 6.633 \\
\hline $3 d 6 p$ & 7.033 & 7.02 & 7.038 & 7.080 \\
\hline $3 d 7 p$ & 7.397 & 7.39 & 7.342 & 7.415 \\
\hline $3 d 8 p$ & 7.465 & 7.47 & 7.471 & 7.502 \\
\hline $3 d 9 p$ & 7.551 & - & 7.556 & 7.575 \\
\hline $3 d 10 p$ & 7.610 & - & 7.614 & 7.624 \\
\hline $4 p 5 s$ & 7.159 & 7.13 & 7.166 & 7.300 \\
\hline $3 d 4 f$ & 6.937 & - & 6.938 & 6.960 \\
\hline $3 d 5 f$ & 7.240 & 7.25 & 7.248 & 7.260 \\
\hline $3 d 6 f$ & 7.425 & - & 7.427 & 7.427 \\
\hline $3 d 7 f$ & 7.523 & - & 7.529 & 7.527 \\
\hline $3 d 8 f$ & 7.591 & - & 7.596 & 7.593 \\
\hline${ }^{1} P$ & $\Gamma \mathrm{eV}$ & $\Gamma \mathrm{eV}[52]$ & $\Gamma \mathrm{eV}[53]$ & $\Gamma \mathrm{eV}[54]$ \\
\hline $3 d 5 p$ & 0.0801 & 0.21 & 0.0702 & 0.0846 \\
\hline $3 d 6 p$ & 0.0059 & 0.17 & 0.0056 & 0.0067 \\
\hline $3 d 7 p$ & 0.0451 & - & 0.0509 & 0.0399 \\
\hline $3 d 8 p$ & 0.0261 & 0.14 & 0.0232 & 0.0315 \\
\hline $3 d 9 p$ & 0.0163 & - & 0.0141 & 0.0282 \\
\hline $3 d 10 p$ & 0.0140 & - & 0.0101 & 0.0207 \\
\hline $4 p 5 s$ & 0.0129 & 0.15 & 0.0139 & 0.0132 \\
\hline $3 d 4 f$ & 0.00006 & - & 0.000004 & 0.00001 \\
\hline $3 d 5 f$ & 0.0059 & - & 0.0028 & 0.00003 \\
\hline $3 d 6 f$ & 0.0019 & 0.17 & 0.0014 & 0.0024 \\
\hline $3 d 7 f$ & 0.0009 & - & 0.0011 & 0.00007 \\
\hline $3 d 8 f$ & 0.00007 & - & 0.00008 & 0.00006 \\
\hline
\end{tabular}

\section{Conclusions}

The method of interacting configurations in the complex number representation, which was applied earlier to the description of quasi stationary states of a helium atom [1-3], is under consideration. The calculation of the ionization processes for more complicated atomic systems is suggested. New results as well as the brief review of our previous investigations are presented. In the problem of the electronimpact ionization the spectroscopic characteristics of the lowest AIS of $\mathrm{Be}, \mathrm{Mg}, \mathrm{Ca}$ atoms and $\mathrm{H}^{-}, \mathrm{Li}^{+}, \mathrm{Be}^{++}$ions were studied. The energies and the widths of the lowest AIS $\left({ }^{1} S,{ }^{1} P,{ }^{1} D,{ }^{1} F\right)$ of $\mathrm{Be}, \mathrm{Mg}$ atom, together with the lowest $\left({ }^{1} P\right)$ AIS of $\mathrm{Ca}$ atom and $\mathrm{Li}^{+}, \mathrm{Be}^{++}$ions, were calculated. Lowest quasi stationary states of $\mathrm{H}^{-}$ion were studied as well. The found results were compared with known experimental data and calculations on the basis of other methods. Hence, we may draw conclusion about a successful verification of the ICCNR method for the calculation of AIS of complex atoms and the processes of electron-impact ionization and excitation of such atoms.

\section{REFERENCES}

[1] S.M. Burkov, N.A. Letyaev, S.I. Strakhova, T.M. Zajac. Photon and electron ionization of helium to the $n=2$ state of $\mathrm{He}^{+}$, J. Phys. B: At. Mol. Opt. Phys., Vol.21, No.7, 1195-1208 (1988).

[2] S.M. Burkov, T.M. Zajac, S.I. Strakhova. Ionization of helium by fast electrons in the region over the threshold of exiting ions formation, Opt. Spektrosk., Vol.63, No.3, 17-25 (1988).

[3] S.M. Burkov, S.I. Strakhova, T.M. Zajac. Total and partial generalized oscillator strengths for transitions to the continuum of helium, J. Phys. B: At. Mol. Opt. Phys., Vol.23, No.20, 3677-3690 (1990)

[4] T.M. Zajac, V.M. Simulik. The method of interacting configurations in complex number representation. Application for calculations of spectroscopic characteristics of quasistationary states in two electron systems, Int. J. Pure. Appl. Phys., Vol.3, No.3, 243-260 (2007).

[5] T.M. Zajac, A.I. Opachko, V.M. Simulik. Photoionization of $\mathrm{Li}^{+}$ion above the excited ion formation threshold. Book of Abstracts of 4-th CEPAS Conference, Cluj-Napoca, Romania, 18-20 June, 2008, p. 139.

[6] T.M. Zajac, A.I. Opachko, V.M. Simulik. Application of the method of interacting configurations in complex number representation to the problem of the ionization of ions, Uzhgorod Univ. Sci. Herald, Ser. Phys., Vol.22, No.1, 82-86 (2008) (in ukrainian).

[7] A.I. Opachko, V.M. Simulik, R.V. Tymchyk, T.M. Zajac. Lowest autoionizing states of beryllium in photoionization problem. Book of abstracts of 43-th EGAS Conference, University of Fribourg, Fribourg, Switzerland, 28 June-2 July, 2011, p. 203.

[8] T.M. Zajac, A.I. Opachko, V.M. Simulik, R.V. Tymchyk. Ionization of $\mathrm{Mg}$ atom by electron-impact in the region of autoionizing states excitation. Book of abstracts of 41-th EGAS Conference, University of Gdansk, Gdansk, Poland, 8-10 July, 2009, p. 156.

[9] R.V. Tymchyk, T.M. Zajac, V.M. Simulik, A.I. Opachko. Calculation of energetic positions of the lowest autoionizing states of $\mathrm{Mg}$ atom. Abstracts of 10-th European Conference on Atoms Molecules and Photons, Salamanca, Spain, 4-10 July, 2010, Electron collisions, P-055. 
[10] V.M. Simulik, R.V. Tymchyk, T.M. Zajac. Autoionizing states of $\mathrm{Ca}$ in the problem of ionization of calcium atom by the electrons. Book of abstracts of 44-th EGAS Conference, University of Gothenburg, Gothenburg, Sweden, 9-13 July, 2012, p. 197.

[11] V.M. Simulik, T.M. Zajac, R.V. Tymchyk. Calculations for electron-impact ionization of beryllium in the method of interacting configurations in the complex number representations, arXiv: 1608.04078v1 [physics, atom-ph] 14 Aug. 2016. 5 p.

[12] T.M. Zajac, V.M. Simulik, R.V. Tymchyk. The beryllium atom lowest autoionizing states in the method of interacting configurations in the complex number representations, Int. J. Theor. Math. Phys., Vol.6, No.4, 111-116 (2016).

[13] V.M. Simulik, T.M. Zajac, R.V. Tymchyk. Application of the method of interacting configurations in the complex number representation to calculating the spectroscopic characteristics of the autoionizing states of $\mathrm{Be}, \mathrm{Mg}$ and $\mathrm{Ca}$ atoms, Ukr. J. Phys., Vol.60, No.11, 1094-1100 (2015).

[14] V.M. Simulik, T.M. Zajac, R.V. Tymchyk. Choice of the wave function for the helium ground state for precision calculations of quasi stationary state parameters, Ukr. J. Phys., Vol.61, No.11, 950-955 (2016).

[15] G. Mehlman-Ballofet, J.M. Esteva. Far ultraviolet absorption spectra with auto-ionized levels of beryllium and magnesium, Astrophys. J., Vol.157, No.2, 945-956 (1969).

[16] J.M. Esteva, G. Mehlman-Ballofet, J. Romand. Spectres d'absorption dans l'ultraviolet lointain de Be, B, C, N, Mg, $\mathrm{Al}$ et $\mathrm{Si}$, Journal of Quantitative Spectroscopy and Radiative Transfer, Vol.12, No.9, 1291-1303 (1972).

[17] P.L. Altic. Photo-ionization cross section of beryllium near threshold, Phys. Rev., Vol.169, No.1, 21-26 (1968).

[18] V.L. Lengyel, V.T. Navrotsky, E.P. Sabad. Resonant scattering of low-energy electrons by $\mathrm{Be}^{+}$and $\mathrm{Mg}^{+}$ions, J. Phys. B: At. Mol. Opt. Phys., Vol.23, No.16, 2847-2867 (1990).

[19] H.C. Chi, K.N. Huang, K.T. Cheng. Autoionizing levels of beryllium from the multiconfiguration relativistic random-phase approximation, Phys. Rev. A., Vol.43, No.5, 2542-2545 (1991).

[20] D.S. Kim, S.S. Tayal, H.L. Zhou, S.T. Manson. Photoionization of atomic beryllium from the ground state, Phys. Rev. A., Vol.61, No.6, 062701-062709 (2000).

[21] T.M. Clark et al. Observation of autoionizing states of beryllium by resonance-ionization mass spectrometry, J. Opt. Soc. Am. B., Vol.2, No.6, 891-896 (1985).
[22] R Moccia, P Spizzo. One-photon transition probabilities and photoionisation cross section calculations of Be, J. Phys. B: At. Mol. Phys., Vol.18, No.17, 3537-3554 (1985).

[23] H. Bachau, P. Galan, and F. Martin. Feshbach-model potential approach for the study of resonant and bound states of Be-like ions, Phys. Rev. A., Vol.41, No.7, 3534-3544 (1990).

[24] N. Miura, Y. Osanai, T. Noro, F. Sasaki. Theoretical determination of energies and widths of autoionizing states of the Be atom, J. Phys. B: At. Mol. Opt. Phys., Vol.29, No.13, 26892699 (1996).

[25] K. Bartschat, P.G. Burke, M.P. Scott. Electron-impact excitation of beryllium, J. Phys. B: At. Mol. Opt. Phys., Vol.29, No.20, L769-L772 (1996).

[26] J. Colgan et al. Electron-impact ionization of all ionization stages of beryllium, Phys. Rev. A., Vol.68, No.3, 032712(1-9) (2003).

[27] R. Wehlitz, D. Lukic, J. B. Bluett. Resonance parameters of autoionizing Be $2 p n l$ states, Phys. Rev. A., Vol.68, No.5, 052708(1-5) (2003).

[28] C.P. Ballance et al. Electron-impact excitation of beryllium and its ions, Phys. Rev. A., Vol.68, No.6, 062705(1-11) (2003).

[29] M.A. Uddin et al. Electron-impact ionization of beryllium isoelectronic ions, Int. J. Mass. Spectr., Vol.244, No.1, 76-83 (2005).

[30] J.C. Cardona, J.L. Sanz-Vicario. Autoionizing states in beryllium-like atomic systems using explicitly correlated coordinates, J. Phys. B: At. Mol. Opt. Phys., Vol.41, No.5, 055003(1-10) (2008).

[31] M.R. Talukder. Electron impact total ionization cross sections of beryllium and boron isoelectronic ions, Appl. Phys. B., Vol.93, No.2-3, 567-574 (2008).

[32] W.C. Chu, C.D. Lin. Theory of ultrafast autoionization dynamics of Fano resonances, Phys. Rev. A., Vol.82, No.5, 053415(1-9) (2010).

[33] M.S. Pindzola, C.P. Ballance, F. Robicheaux, J. Colgan. Electron-impact double ionization of beryllium, J. Phys. B: At. Mol. Opt. Phys., Vol.43, No.10, 105204(1-5) (2010). 
[34] J.M.N. Djiokap, A.F. Starace. Resonant enhancement of the harmonic generation spectrum of beryllium, Phys. Rev. A., Vol.88, No.5, 053412(1-15) (2013).

[35] T. Maihom et al. Electron-impact ionization cross sections of beryllium and beryllium hydrides, Eur. Phys. J. D., Vol.67, No.1, 2(1-5) (2013).

[36] O. Zatsarinny, K. Bartschat, D.V. Fursa. Calculations for electron-impact excitation and ionization of beryllium, J. Phys. B: At. Mol. Opt. Phys., Vol.49, No.23, 235701(1-9) (2016).

[37] M.A. Baig, J.P. Connerade. Extensions to the Spectrum of Doubly Excited MgI in the Vacuum Ultraviolet, Proc. Roy. Soc. Lond. A., Vol.364, December 20, 353-366 (1978).

[38] D.S. Kim, S.S. Tayal. Autoionizing resonances in the photoionization of ground state atomic magnesium, J. Phys. B: Atom. Mol. Opt. Phys., Vol.33, No.17, 3235-3247 (2000).

[39] Y.L. Shao, C. Fotakis, D. Charalambidis. Multiphoton ionization of $\mathrm{Mg}$ in the wavelength region of 300-214 nm, Phys. Rev. A., Vol.48, No.5, 3636-3643 (1993).

[40] M.J. Ford et al. Electron-impact double ionization of magnesium, Phys. Rev. A., Vol.57, No.1, 325-330 (1998).

[41] K. Bartschat, D. Weflen, X. Guan. Electron-impact ionization of magnesium, J. Phys. B: Atom. Mol. Opt. Phys., Vol.40, No.16, 3231-3239 (2007).

[42] T.K. Fang, Y.K. Ho. Determination of resonance energies and widths of $\mathrm{Mg} 3 p n l{ }^{1} \mathrm{D}^{e}$ and ${ }^{1} \mathrm{~F}^{0}$ doubly excited states by the stabilization method with the B-spline-based configuration interaction approach, J. Phys. B: Atom. Mol. Opt. Phys., Vol.32, No.15, 3863-3872 (1999).

[43] A. Reber, F. Martin, H. Bachau, R.S. Berry. Two-photon above-threshold ionization of magnesium, Phys. Rev. A., Vol.65, No.6, 063413(1-7) (2002).

[44] A. Reber, F. Martin, H. Bachau, R.S. Berry. Three-photon above-threshold ionization of magnesium, Phys. Rev. A., Vol.68, No.6, 063401(1-10) (2003).

[45] A. Reber et al. Above-threshold ionization near the $3 p 4 d{ }^{1} F^{0}$ autoionizing state in magnesium, Phys. Rev. A., Vol.71, No.5, 053402(1-7) (2005).
[46] D. Rassi, V. Pejcev, T.W. Ottley, K.J. Ross. High-resolution ejected-electron spectrum of magnesium autoionising levels following two-electron excitation by low-energy electron impact, J. Phys. B: Atom. Mol. Phys., Vol.10, No.14, 2913-2921 (1977).

[47] T.M. El-Sherbini, A.A. Rahman. Autoionizing states in MgI, Annalen der Phys., Vol.39, No.5, 333-337 (1982).

[48] T.N. Chang. 3pnp ${ }^{1} S$ autoionization states of the magnesium atom, Phys. Rev. A., Vol.36, No.11, 5468-5470 (1987).

[49] C.J. Dai. Resonance profiles of Mg 3pns autoionizing states, Chin. Phys. Lett., Vol.12, No.3, 152-155 (1995).

[50] R.F. Boivin, S.K. Srivastava. Electron-impact ionization of Mg, J. Phys. B: Atom. Mol. Opt. Phys., Vol.31, No.10, 2381-2394 (1998).

[51] J.A. Ludlow et al. Electron-impact single ionization of $\mathrm{Mg}$ and $\mathrm{Al}^{+}$, Phys. Rev. A., Vol.79, No.3, 032715(1-6) (2009).

[52] J.E. Kontrosh, I.V. Chernyshova, O.B. Shpenik. Nearthreshold electron-impact ionization of calcium atom, Opt.Spectrosc., Vol.110, No.4, 500-507 (2011).

[53] O.I. Zatsarinny, V.I. Lengyel, E.A. Masalovich. Resonance structure in the electron-impact excitation of $\mathrm{Ca}^{+}$below the $5 s$ threshold, Phys. Rev. A., Vol.44, No.11, 7343-7354 (1991).

[54] P. Scott, A.E. Kingston, A. Hibbert. Photoionization of neutral calcium, J. Phys. B: Atom. Mol. Phys., Vol.16, No.21, 3945-3959 (1983).

[55] S.M. Kazakov, O.V. Khristoforov. Electron spectra from autoionizing states of strontium and calcium excited by low and intermediate-energy electrons, Sov. Phys. JETP, Vol.61, No.4, 656-664 (1985)

[56] D. Cvejanovic, A.J. Murray. Single ionization of calcium by electron impact, J. Phys. B: Atom. Mol. Opt. Phys., Vol.36, No.17, 3591-3605 (2003).

[57] D. Cvejanovic, A. J. Murray. Single ionization of calcium by electron impact, J. Phys. B: Atom. Mol. Opt. Phys., Vol.36, No.8, 3591-3605 (2003).

[58] D. Ma, N. Zheng, J. Fan. Theoretical analysis on $3 d n l$ $J=1^{e}-5^{e}$ autoionizing levels in Ca, J. Phys. Chem. Ref. Data, Vol.33, No.4, 1013-1030 (2004). 
[59] M.E. Hamm et al. Observation of narrow resonances in the $\mathrm{H}^{-}$photodetachment cross section near the $\mathrm{n}=3$ threshold, Phys. Rev. Lett., Vol.43, No.23, 1715-1718 (1979).

[60] R.S. Oberoi. Auto-ionization states of two electron atoms, J. Phys. B: Atom. Mol. Phys., Vol.5, No.6, 1120-1125 (1972).

[61] K.T. Chung. Projection-operator approximation for autoionization states in the inelastic scattering region, Phys. Rev. A., Vol.6, No.5, 1809-1813 (1972).

[62] C.D. Lin. Feshbach and shape resonances in the $e-\mathrm{H}^{1} P$ system, Phys. Rev. Lett., Vol.35, No.17, 1150-1153 (1975).

[63] L.A. Morgan, M.R.C. McDowell, J. Callaway. Electronimpact excitation of resonances below the $n=3$ levels of atomic hydrogen, J. Phys. B: Atom. Mol. Phys., Vol.10, No.16, 3297-3305 (1977).

[64] B.L. Christensen-Dalsgaard. Two-electron correlation: diabatic states, Phys. Rev. A., Vol.29, No.2, 470-487 (1984).

[65] A. Macias, A. Riera. Use of a simple method for the resonance widths of ${ }^{1} P$ states in He-like systems, Europhys. Lett., Vol.2, No.5, 351-358 (1986).

[66] A.G. Abrashkevich et al. Doubly excited states of $\mathrm{H}^{-}$and $\mathrm{He}$ in the coupled-channel hyperspherical adiabatic approach, Phys. Rev. A., Vol.45, No.7, 5274-5277 (1992).

[67] A.G. Abrashkevich, M. Shapiro. Theory of two-electron atoms interacting with intense laser pulses: the one-photon ionization of $\mathrm{He}$ and the photodetachment of $\mathrm{H}^{-}$, J. Phys. B: Atom. Mol. Opt. Phys., Vol.29, No.4, 627-644 (1996).

[68] A. Burgers, E. Lindroth. Doubly excited states in the negative hydrogen ion, Eur. Phys. J. D., Vol.10, No.2, 327-340 (2000).

[69] M. Dieng, I. Sakho, M. Biaye, A. Wague. The Intra-shell singlet and triplet doubly excited states energy calculations of helium-like Ions using special forms of the Hylleraas-type wave functions, Chin. J. Phys., Vol.48, No.1, 38-48 (2010).

[70] R. Bruch, G. Paul, J. Andra, L. Lipsky. Antoionization of foil-excited states in Li I and Li II, Phys. Rev. A., Vol.12, No.5, 1808-1824 (1975).

[71] Y.K. Ho. Autoionization states of helium isoelectric sequence below the $n=3$ hydrogenic thresholds, J. Phys. B: At. Mol. Opt. Phys., Vol.12, No.3, 387-400 (1979).
[72] A. Wague. Application of the diagonalization approximation to the $n=3$ resonant photoionization of helium-like systems, Z. Phys D. At. Mol. Clust., Vol.6, No.4, 337-344 (1987).

[73] Y.K. Ho. Complex-coordinate calculations for doubly excited states of two-electron atoms, Phys. Rev., Vol.23, No.5, 2137-2149 (1981).

[74] K. Berrington, S. Nakazaki. Direct and indirect ionization processes near the Li "hollow-atom" threshold, J. Phys. B: Atom. Mol. Opt. Phys., Vol.31, No.2, 313-327 (1998).

[75] S. Diehl et al. New high-resolution measurements of doubly excited states of $\mathrm{Li}^{+}$, J. Phys. B: Atom. Mol. Opt. Phys., Vol.32, No.17, 4193-4207 (1999).

[76] N. Koyama, A. Takafuji, M. Matsuzawa. High-lying doubly excited states of $\mathrm{H}^{-}$and He: II. ${ }^{1} \mathrm{P}^{0}$ states. J. Phys. B: Atom. Mol. Opt. Phys., Vol.22, No.4, 553-558 (1989).

[77] S.I. Themelis. High-lying doubly excited states of $\mathrm{He}$ and $\mathrm{H}^{-}$: electron correlations and classification schemes, Cent. Eur. J. Phys., Vol.3, No.3, 324338 (2005).

[78] J.C. Ball, C.Y. Chen, D.Y. Wong. Faddeev equations for atomic problems and solutions for the (e,H) system, Phys. Rev., Vol.173, No.1, 202-213 (1968).

[79] V.N. Mileev, V.S. Senashenko, E.Yu. Tsymbal. On the theory of resonance ionization at atoms by fast protons, J. Phys. B: Atom. Mol. Opt. Phys., Vol.14, No.15, 2625-2633 (1981).

[80] A.A. Kvitsinsky, A. Wu, C.Y. Hu. Scattering of electrons and positrons on hydrogen using the Faddeev equations, J. Phys. B: Atom. Mol. Opt. Phys., Vol.28, No.2, 275-285 (1995).

[81] L. D. Faddeev, S. P. Merkuriev. Quantum scattering theory for several particle systems, Kluver, Dordrech, 1993.

[82] E. Nielsen, D.V. Fedorov, A.S. Jensen, E. Garrido. The three body problems with short-range interactions, Phys. Rep., Vol.347, No.5, 373-459 (2001).

[83] U. Fano. Effects of configuration interaction on intensities and phase shifts, Phys. Rev., Vol.124, No.6, 1866-1878 (1961).

[84] J. W. Cooper, U. Fano, F. Prats. Classification of two-electron excitation levels of helium, Phys. Rev. Lett, Vol.10, No.12, 518-521 (1963) 
[85] U. Fano, J. W. Cooper. Line profiles in the far-uv absorption spectra of the rare gases, Phys. Rev., Vol.137, No.5A, 1364-1379 (1965).

[86] U. Fano. Correlations of two excited electrons, Rep. Prog. Phys., Vol.46, No.2, 97-165 (1983).

[87] V.V. Balashov, V.B. Beliaev, R.A. Eramjian, N.M. Kabachnik. Resonance mechanism of neutron emission in muon capture reaction in oxygen, Phys. Lett., Vol.9, No.2, 168-170 (1964).

[88] V.V. Balashov, G.Ya. Korenman, V.L. Korotkin, V.N. Fetisov. Excitation of the giant dipole resonance in $\mu$-capture, Nucl. Phys. B., Vol.1, No.3, 158-168 (1967).

[89] V.V. Balashov, S.I. Grishanova, I.M. Kruglova, V.S. Senashenko. Possibilities of diagonalization method for calculating the atomic system resonances, Phys. Lett. A., Vol.27, No.2, 101-102 (1968).

[90] V.V. Balashov, S.I. Grishanova. Isobaric analogue resonance as a collective effect of strong coupling of channels, Phys. Lett. B., Vol.28, No.3, 157-158 (1968).

[91] V.V. Balashov, A.N. Grum-Grzhimailo, N.M. Kabachnik. Polarization and correlation phenomena in atomic collisions, Springer-Verlag, Berlin, 2000.

[92] V.V Balashov, S.S Lipovetsky, V.S Senashenko. On the shape of resonance lines in the spectra of electrons ejected from atoms by fast particles, Phys. Lett. A., Vol.40, No.5, 389-390 (1972).

[93] V.I. Lengyel, V.T. Navrotsky, E.P. Sabad. Resonance phenomena in electron-atom collisions, Springer-Verlag, Berlin, 1992.

[94] B.W Shore. Scattering theory of absorption line-profiles and refractivity, Rev. Mod. Phys., Vol.39, No.2, 439-462 (1967).

[95] B.W Shore. The theory of coherent atomic excitation, Vol.1,2, John Wiley and Sons, New York, 1990.

[96] P.G. Burke, A. Hibbert, W.D. Robb. Electron scattering by complex atoms, J. Phys. B: Atom. Mol. Phys., Vol.4, No.2, 153-161 (1971).

[97] K.A. Berrington, P.G. Burke, W.C. Fon, K.T. Taylor. Photoionisation of helium, J. Phys. B: Atom. Mol. Phys., Vol.15, No.17, L603-L608 (1982).

[98] V.P. Jigunov, B.N. Zaharyev. Closed coupled channels methods in quantum scattering theory, Atomizdat, Moscow, 1974 (in russian).
[99] P. McGuire, D.J. Kouri. Quantum mechanical close coupling approach to molecular collisions. $j_{z}$-conserving coupled states approximation, J. Chem. Phys., Vol.60, No.6, 2488-2499 (1974).

[100] R.G. Parr, W. Yang. Density-functional theory of atoms and molecules, Oxford university press, New York, 1989.

[101] E.P. Wigner, L. Eisenbud. Higher angular mornenta and long range interaction in resonance reactions, Phys. Rev., Vol.72, No.1, 29-41 (1947).

[102] P.G. Burke. R-matrix theory of atomic collisions, Springer, New York, 2011.

[103] O.I. Zatsarinny, K. Bartschat. The B-spline R-matrix method for atomic processes: application to atomic structure, electron collisions and photoionization, J. Phys. B: At. Mol. Opt. Phys., Vol.46, No.11, 112001(1-39) (2013).

[104] D.E. Ramaker, D.M. Schrader. Multichannel configurationinteraction theory: Application to some resonances in helium, Phys. Rev. A., Vol.9, No.5, 1980-1991 (1974).

[105] H. Feshbach. Unified theory of nuclear reactions, Ann. Phys., Vol.5, No.4, 357-390 (1958).

[106] C. Bloch. Une formulation unifie de la thorie des ractions nuclaires, Nucl. Phys., Vol.4, No.1, 503-528 (1957).

[107] P. Bogdanovich, R. Karpukiene. The theoretical study of the overlapping configurations $2 \mathrm{~s} 2 \mathrm{p}^{5} 3 l$ and $2 \mathrm{~s}^{2} 2 \mathrm{p}^{4} 4 l$ in Ar X, J. Phys. B: At. Mol. Opt. Phys., Vol.38, No.10, 1557-1568 (2005).

[108] R.K. Peterkop. Theory of ionization of atoms by electron impact, Zinatne, Riga, 1975 (in russian).

[109] V.A. Khyr et al. Cluster spectroscopic factors in the Kharmonics method, Phys. Lett. B., Vol.76, No.3, 273-276 (1978).

[110] W. Bian, X. Zhao, Y. Wang, Y. Wang. Direct solution of the Schrdinger equation for some muonic molecules, Chem. Phys., Vol.242, No.2, 195-202 (1999).

[111] E. Nielsen, D.V. Fedorov, A.S. Jensen, E. Garrido. The three-body problem with short-range interactions, Phys. Rep., Vol.347, No.5, 373-459 (2001).

[112] J.A.C. Alcaras. On the first approximation of the Kharmonics method, J. Math. Phys., Vol.19, No.1, 1-4 (1978). 
[113] A.G. Abrashkevich, M.S. Kaschiev, S.I. Vinitsky. A new method for solving an eigenvalue problem for a system of three Coulomb particles within the hyperspherical adiabatic approach, J. Comput, Phys., Vol.163, No.2, 328-348 (2000).

[114] C. Froese Fischer. The Hartree-Fock method for atoms - a numerical approach, John Wiley and Sons Inc., New York, 1977.

[115] C. Froese Fischer. A general multi-configuration HartreeFock program, Comp. Phys. Comm, Vol.14, No.1-2, 145-153 (1978).

[116] C. Froese Fischer. The MCHF atomic-structure package, Comp. Phys. Comm, Vol.128, No.3, 635-636 (2000).

[117] M.Ya. Amusia. Atomic photoeffect, Springer-Verlag, Berlin, 1990.

[118] M.Ya. Amusia, A.S. Kheifets. The influence of "twoelectron-two-hole"' excitations on the $3 s^{-1} 4 p$ autoionization profile in Ar atoms, Phys. Lett. A., Vol.82, No.8, 407-411 (1981).
[119] Y.K. Ho. Doubly excited ${ }^{1} P^{0}$ resonances in He, Z. Phys. D., Vol.21, No.3, 191-196 (1991).

[120] Y.K. Ho. Doubly-excited ${ }^{1,3} P^{0}$ autoionization states of He between the $\mathrm{N}=4$ and $\mathrm{N}=6$ thresholds of $\mathrm{He}^{+}$ions, Z. Phys. D., Vol.42, No.2, 77-81 (1997).

[121] Y.K. Ho, Z-C. Yan. Calculation of the $\mathrm{He}^{-} 1 \mathrm{~s} 2 \mathrm{~s}^{22} \mathrm{~S}$ resonance using fully correlated Hylleraas functions, Phys. Rev. A., Vol.59, No.4, R2559-R2562 (1999).

[122] L. G. Jiao, Y.K. Ho. Complete supermultiplet structures for the doubly excited intrashell resonances of $\mathrm{H}^{-}$associated with the $\mathrm{H}(\mathrm{N}=2,3$, and 4) thresholds, Phys. Rev. A., Vol.89, No.5, 052511(1-9) (2014).

[123] E.A. Hylleraas. Uber den Grundzustand des Heliumatoms, Z. Phys., Vol.48, No.7-8, 469494 (1928).

[124] R.J. Tweed. Correlated wavefunctions for helium-like atomic systems, J. Phys. B: At. Mol. Phys., Vol.5, No.4, 810-819 (1972). 